Tohoku J. Exp. Med., 2008, 215, 79-87

\title{
Expression of MHC-I mRNA in Peripheral Blood Lymphocytes as an Early Marker of Acute Rejection Following Skin Transplantation in Mice
}

\author{
Furong Wan, ${ }^{1}$ Nan Lu, ${ }^{1}$ Xiong Zou, ${ }^{1}$ Yi Zhang, ${ }^{1}$ Ningning Shan, ${ }^{1}$ \\ Xiaojing Yang, ${ }^{1}$ Xiangdong Li, ${ }^{2}$ Shengmei Zhao, ${ }^{1}$ Shenghua Wang, ${ }^{1}$ \\ Mingchen Zhu, ${ }^{1}$ Xiaoli Li, ${ }^{1}$ Yinguie Li, ${ }^{1}$ YuXia Zhou ${ }^{1}$ and $\mathrm{Hao} \mathrm{Li}^{1}$ \\ ${ }^{1}$ Department of Clinical Laboratory, Qilu Hospital, Shandong University, Jinan, P.R. China \\ ${ }^{2}$ Department of Clinical Laboratory, Qianfoshan Hospital, Jinan, P.R. China
}

\begin{abstract}
Early prediction of acute rejection (AR) is important in clinical practice of organ and tissue transplantation. The aims of this study were to investigate the expression of major histocompatibility complex (MHC)-I and MHC-II genes in peripheral blood lymphocytes (PBLs) following skin grafting and whether their expression can be used as early markers for AR. Skin-grafted mice were selected as an animal model and PBL samples were collected daily for up to 2 weeks post-transplant. Full-thickness skin from the backs of C57BL/6 mice (H-2b) was transplanted onto that of BALB/c mice $(\mathrm{H}-2 \mathrm{~d})$ in allograft group $(\mathrm{H}-2 \mathrm{~b}$ to $\mathrm{H}-2 \mathrm{~d})$ and in syngeneic graft group $(\mathrm{H}-2 \mathrm{~d}$ to $\mathrm{H}-2 \mathrm{~d})$. The expression levels of MHC-I (H-2K, H-2D) and MHC-II (H-2Ia, H-2Ie) mRNAs were examined using realtime PCR. The histopathological changes of graft biopsies were also analyzed with hematoxylin-eosin staining. The real-time PCR analysis showed that MHC-I and MHC-II mRNA levels were increased in a bimodal distribution pattern during AR in allograft group, whereas no significant changes were detected in syngeneic graft group. The level of $\mathrm{H}-2 \mathrm{~K}$ mRNA was significantly increased at day 5 post-transplants compared with those pre-transplant controls $(p<0.01)$. This increase was detected 5-6 days earlier before graft rejection observed macroscopically. H-2K mRNA level was increased significantly in $93.8 \%$ of mice $(61 / 65)$ in allograft group. These results indicate that the expression of MHC-I and MHC-II mRNAs is up-regulated in PBLs during AR. Especially, the expression of $\mathrm{H}-2 \mathrm{~K}$ mRNA can be used as an early marker for AR. —— MHC; PBLs; acute rejection; transplantation; gene expression.
\end{abstract}

Tohoku J. Exp. Med., 2008, 215 (1), 79-87.

(C) 2008 Tohoku University Medical Press

Acute rejection (AR) is one of the major contributors to allograft loss. It is well-known that earlier detection of $\mathrm{AR}$ is vital for improving the short- or long-term survival of the transplants. Although graft histopathological examination remains the golden standard in the diagnosis of AR, patients are unwilling to accept biopsy because it is invasive and costly. There is an urgent need for the development of more reliable and noninvasive early markers. At present, some

Received January 7, 2008; revision accepted for publication March 22, 2008.

Correspondence: Prof. Xiong Zou, Department of Clinical Laboratory, Qilu Hospital, Shandong University,

107 Wenhua Xi Road, Jinan, 250012, P.R. China.

e-mail: zx2186@21cn.com 
noninvasive monitoring markers, such as peripheral blood lymphocytes (PBLs) CD4/CD8 ratios, interleukin (IL)-2, IL-6, tumor necrosis factor (TNF) cytokines, C-reactive protein, and serum soluble CD30 have been used in clinical practice. However, the sensitivities and specificities of these markers are not satisfactory when used for post-transplant monitoring of immunological status of allograft recipients.

Recently the major histocompatibility complex (MHC) has attracted attention. First, the gene expression of lymphocyte marker HLA-DR (MHC-II), perforin and granzyme B mRNA in PBLs have been proposed as noninvasive markers for kidney transplantation rejection (Sabek et al. 2002). Serum soluble HLA-I (MHC-I) has also been used to detect acute rejection in kidney and liver transplantation (Mathew et al. 1996; Borelli et al. 1999). However, the concentration of soluble HLA-I is very low in blood serum and the quantitative method of enzyme-linked immunosorbent assay (ELASA) can not meet the clinical need in sensitivity and specificity.

Lymphocytes expressed abundant MHC-I molecules that appeared consistently in acutely rejecting transplants. Currently, there was few research about the role of MHC-I gene expression in PBLs during AR. In our study, the non-polymorphic sequence of MHC gene was selected to design primers and the expression levels of MHC-I and -II mRNAs were quantitatively measured using real time PCR during AR. Furthermore, we studied the correlations between the expression of MHC-I and -II mRNAs and the pathological changes of the skin graft. The purpose of this study was to explore the variations of MHC-I mRNA expression level in AR and assess the possibility of its expression as an early marker of AR.

\section{Materials and Methods}

\section{Animal model}

Purified inbred Specific Pathogen Free (SPF) C57BL/6 and BALB/c mice (male, weighing 20-25 g) were obtained from Institute of Zoology of Chinese Academy of Sciences (Beijing, China). The experimental protocol was approved by Ethical Committee of
Shandong University. Animals received a standard diet and water and were cared in accordance with the Principles of Laboratory Animal Care (NIH publication No. 86-23, revised 1985) as well as the China Committee of Animal Experimentation. Full-thickness skin from the backs of C57BL/6 mice ( $\mathrm{H}-2 \mathrm{~b}$, donor) was transplanted onto that of $\mathrm{BALB} / \mathrm{c}$ mice (H-2d, recipient) using standard techniques in allograft group $(\mathrm{H}-2 \mathrm{~b}$ to $\mathrm{H}-2 \mathrm{~d})$ and in syngeneic graft group ( $\mathrm{H}-2 \mathrm{~d}$ to $\mathrm{H}-2 \mathrm{~d})$. Bandages were removed at day 5 post-transplant. Skin grafts were scored twice daily. Each graft was considered rejected when $60 \%$ or more of the graft tissue was destroyed (Silva et al. 2006), by the macroscopic appearance of graft necrosis, followed by histopathological confirmation.

\section{Experimental group}

All mice $(n=160)$ were randomly divided into three groups: control group (pre-transplant healthy individuals, $n=30$ ); allograft group (C57BL/6 to BALB/c, $n=65$ ); syngeneic graft group (BALB/c to BALB/c, $n=65$ ).

\section{Blood sample}

Five mice from each experimental group were sacrificed for peripheral blood samples every day (day 2-14) after skin transplantation. PBLs samples were collected daily for up to 2 weeks post-transplant and were isolated initially from heparinized whole blood by density gradient centrifugation on a lymphocyte separation agent (TBD, Tianjin, China), and deposited in glassware for one hour at room temperature $\left(25^{\circ} \mathrm{C}\right)$. Lymphocytes were obtained from suspended cells.

\section{Biopsies}

Biopsies from the allografts and syngeneic grafts were processed after transplantation. The necrotic tissues caused by inappropriate operation were not chosen in our study. The biopsies were fixed in $4 \%$ formalin solution, embedded in paraffin, and cut into $5-\mu \mathrm{m} \mathrm{sec}-$ tions and stained with hematoxylin-eosin. The fullthickness skin transplants rejection was graded according to Bejarano's approach (Bejarano et al. 2004) as follows. Grade 0: no rejection and no perivascular infiltrates. Grade 1: up to $10 \%$ of vessels showing infiltrates of small lymphocytes. Grade 2: mild rejection with $11 \%$ to $50 \%$ of vessels being infiltrated by small lymphocytes. Eosinophils and mild spongiosis may or may not be present. No epidermal infiltrates, stromal infiltrates or large lymphocytes are seen. Grade 3: moderate rejection with greater than $50 \%$ of vessels showing lymphocyte infil- 
trates that may be accompanied by epidermal and stromal inflammation. Spongiosis is absent or mild. Endothelial plumping, eosinophils, and large lymphocytes may be seen. Grade 4: severe rejection with greater than $50 \%$ of vessels show infiltrates, but different from moderate rejection, there is dyskeratosis and the epidermis shows heavier lymphocyte infiltrates and moderate to severe spongiosis. The stroma shows infiltrates extending into the base of the epidermis. Endothelial plumping or necrosis, eosinophils, and large lymphocytes are present.

\section{RNA extraction and real-time PCR}

Total RNA was extracted from lymphocytes (concentration was $3 \times 10^{6} / \mathrm{ml}$ ) using TRIZOL (Invitrogen Corp., Carlsbad, CA, USA). Total RNA was briefly performed to RNAase-free DNAase I (DNA-free; Ambio Inc., NY, USA) to remove any residual genomic DNA, and $1 \mu \mathrm{g}$ reverse transcribed to cDNA using M-MLV Reverse Transcriptase (Promega, Madison, WI, USA). Primers were designed using Primer Express Software (Applied Biosystems, Foster, CA, USA). Beta-actin was chosen as an internal standard. All primers were obtained from Invitrogen Corp. The expression of MHC-I and -II mRNAs were determined using the Lighter Cycler 2.0 real-time quantitative PCR instrument (Roche, Grenzacherstrasse, Basel, Switzerland). All PCR reagents and protocol were provided by the manufacture from the Sybr-green real-time PCR kit (TaKaRa, Dalian, China). A typical reaction contained $1 \mathrm{ng}$ total RNA and SYBR Premix Ex Taq $^{\mathrm{TM}} 10 \mu \mathrm{l}$ in $20 \mu \mathrm{l}$ reaction volume. Primers were added at a final concentration of $300 \mathrm{nM}$. The PCR reaction consisted of heating at $95^{\circ} \mathrm{C}$ for $10 \mathrm{sec}$, and at $64^{\circ} \mathrm{C}$ for $1 \mathrm{~min}$ and 45 cycles followed by the thermal denaturation protocol. The expression of MHC-I and -II mRNAs compared to beta-actin mRNAs was measured as increased fluorescence, corresponding to amplification and degradation. The cycle number at which the threshold line intersects the linear curve for each sample was used to determine the threshold cycle $\left(C_{T}\right)$ value.

\section{Sample data calculation, statistical analysis}

The relative values of gene amplification were calculated using the equation: $2^{-\triangle \triangle \mathrm{CT}}$ (Jan et al. 2006). For the prediction of rejection, the control samples were chosen for calibration. The post-transplant samples were examined to reflect individual dynamic differences in both immune system activity and response to allograft antigen. All the data were analyzed by SPSS 13.0 (SPSS
Inc., Chicago, IL, USA). The results are shown as mean values \pm S.D. with differences groups. A Shapiro-Wilk's test for normality was performed prior to the analysis of data to allow appropriate parametric or nonparametric statistical tests to be used. Serial measurements were compared using ANOVA techniques with inter-comparison using student's $t$-tests. A $p$ value less than 0.05 was regarded as a significant difference, and less than 0.01 as highly significant.

\section{Results}

\section{Allograft rejection macroscopical analysis}

All mice survived were healthy and gained weight. Each graft was examined daily from day 2 post-transplant and was considered absolutely rejected macroscopically when $60 \%$ or more graft tissue was destroyed (Fig. 1). The absolute rejection was firstly macroscopically presented averagely at day 11 after transplantation in allograft group. Syngeneic grafted controls grew well and no skin rejection was observed.

\section{Pathological analysis of allograft rejection}

Pathological evidence of acute grafts rejection demonstrated severe lymphocytes and monocytes infiltration in the epidermis in allograft group. On the other hand, no obvious infiltration was observed in syngeneic graft group (Fig. 2). In allograft group, grade 0-1 of AR appeared from day 2 to 4 post-transplant, grade 2 appeared from day 5 to 7 , grade 3 appeared from day 8 to 12 and grade 4 (severe rejection) appeared from day 13 to 14.

\section{Analysis of PBLs MHC-I and -II mRNA expres- sion}

Based on the standard and validated assay, we investigated the relationship between the target gene expression, histopathological evidence and macroscopical presence in AR. MHC-I and -II mRNAs expression levels did not show any obvious changes in the syngeneic graft group (Fig. 3, Table 1). However, MHC-I and -II mRNAs levels were elevated, showing bimodal distribution pattern in accordance with AR in allograft group (Fig. 4).

MHC-I mRNA expression increased signifi- 


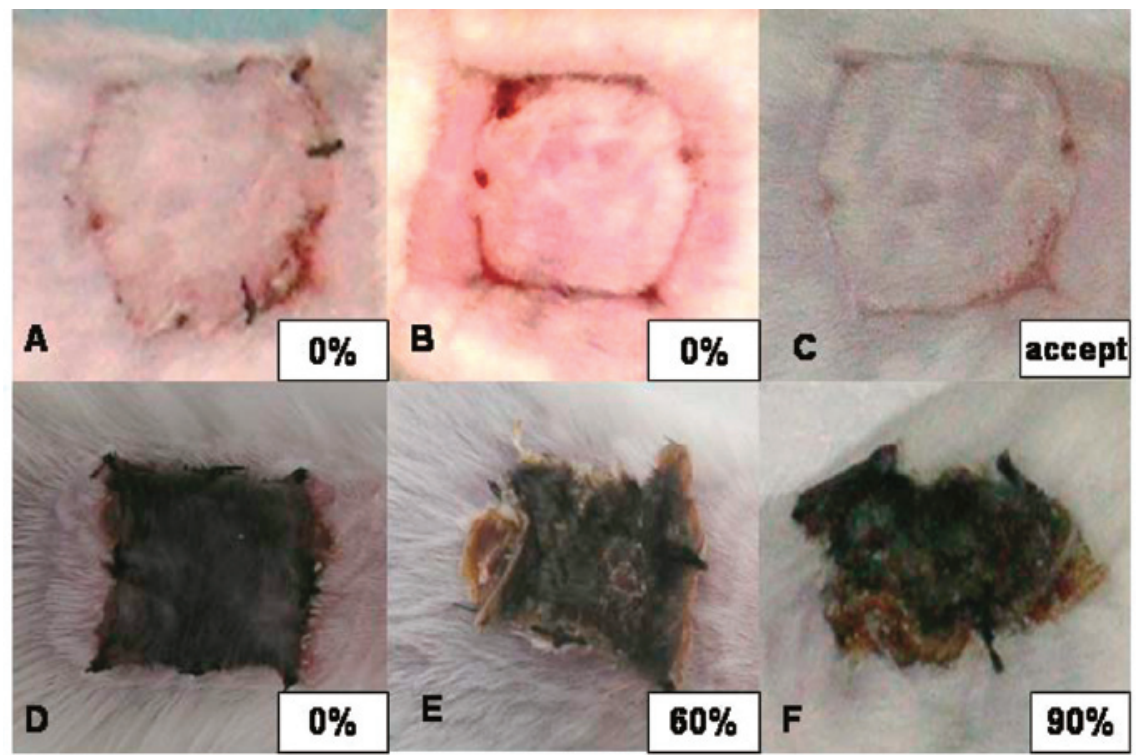

Fig. 1. Syngeneic and allogeneic graft rejection process in gross.

The percentage of skin-graft necrosis was shown. Syngeneic graft was not rejected and grew well (A to C). Allogeneic graft appeared to be acutely rejected and showed skin necrosis (D to F). D: day 2 post-transplant, no rejection. E: day 11, showing $60 \%$ destroyed graft. F: day 13 , showing $90 \%$ destroyed graft.

cantly from day 5 post-transplant (corresponding to grade 2) to reach the first peak at day 7 , then slowly decreased, and increased again to form the second peak at day 11 , corresponding to the grade 3-4. Noticeably, the low valley point levels which appeared at day 9 post-transplant were still higher than pre-transplant levels. It was evident that the significantly increased expression of MHC-I mRNA appeared earlier approximately 5 to 6 days than graft rejection manifested macroscopically.

Compared with MHC-II level, MHC-I mRNA expression level was higher and lasted longer (Fig. 4A). The expression levels of two loci of MHC-I gene, $\mathrm{H}-2 \mathrm{~K}$ and $\mathrm{H}-2 \mathrm{D}$, were also analyzed. H-2K mRNA expression seemed higher than H-2D in the first peak (Fig. 4B). About $94 \%$ of allograft samples (61 of 65 ) showed increased expression levels of $\mathrm{H}-2 \mathrm{~K}$ mRNA at post-transplant than pre-transplant level during $\operatorname{AR}(p<0.01)$.

\section{Discussion}

The major target antigen of immune response to allogeneic tissues was MHC molecules on donor cells. Classical MHC-I molecules included
H-2K, H-2D, and MHC-II included H-2Ia, H-2Ie in mice (Klein et al. 1983). MHC-II (HLA-DR) gene expression as a diagnostic marker during AR of kidney and liver (Davenport et al. 1996) allograft have been reported, but there were few studies on the MHC-I mRNA levels in PBLs in animal models of AR. In contrast to previous studies that measured gene expression in peripheral blood samples, we detected MHC-I (H-2K, $\mathrm{H}-2 \mathrm{D})$ and -II (H-2Ia, H-2Ie) mRNAs expression serially in PBLs during the entire process of acute rejection in murine skin transplantation model.

The MHC transcriptional levels were selected to examine because MHC molecules were highly polymorphic and difficult for quantitative comparison among different individuals. The exon 2 and 3 of MHC-I gene were highly polymorphic and related to transplants match. Both exon 4 and exon 5 were non-polymorphic. This non-polymorphic sequence of MHC-I gene were chosen to design primers that was used to quantitatively measure the expression of MHC-I and -II mRNAs with real time PCR. Real time PCR is rapid, hypersensitive, and reproducible with a wide dynamic range. Peripheral blood was cho- 


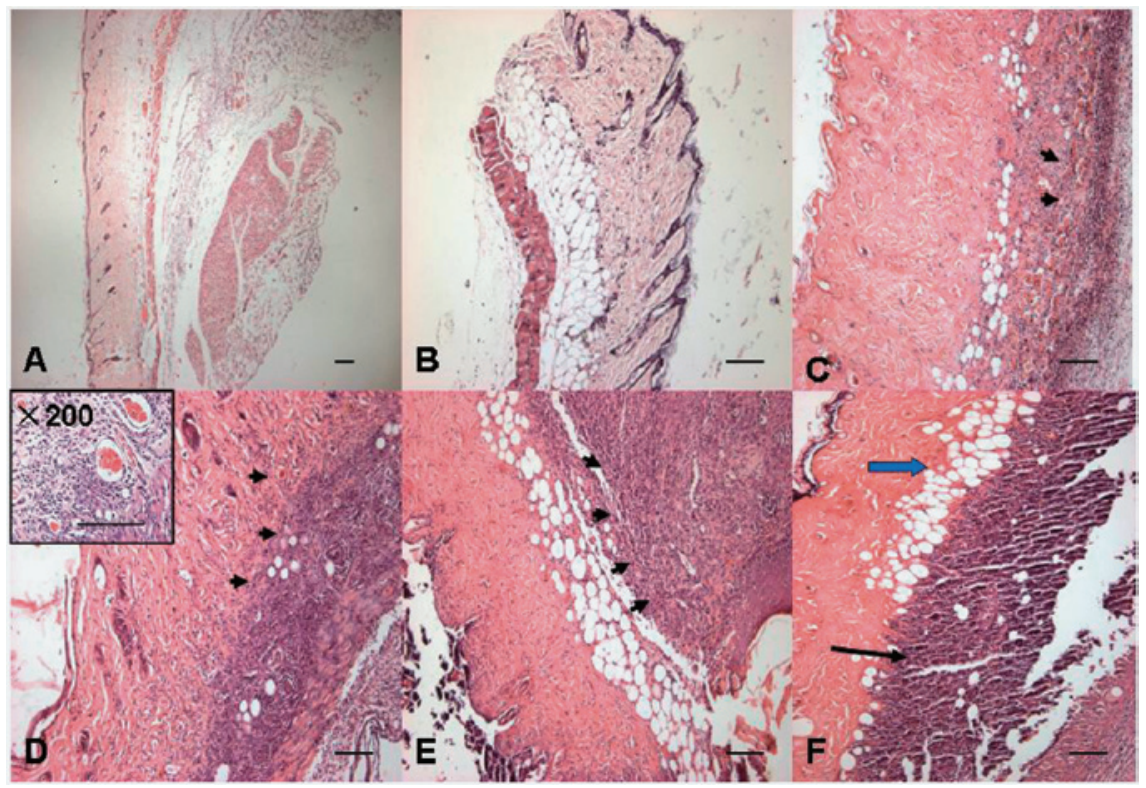

Fig. 2. Pathological evidence in allograft rejection process.

Biopsies from the allografts were performed everyday before and after transplantation and stained with hematoxylin-eosin (H-E). The different grade showing the acute skin graft rejection with the increased lymphocytes and monocytes infiltration gradually in the vessels (panel in D) and epidermis (short black arrow), more spongiosis appearance (blue arrow in F), and necrosis (long black arrow in F) from A to F. A: pre-transplant, grade 0 , no rejection and no infiltrates. B: day 2-4 posttransplant, grade $1,<10 \%$ vessels showing infiltrates. C: day 5-7 post-transplant, grade 2 , mild rejection, $11 \sim 50 \%$ of vessels being infiltrated. D: day 8-10 post-transplant, grade 3, moderate rejection, $>50 \%$ of vessels showing infiltrates; E and F showed day 11-12 and day 13-14 posttransplant respectively, grade 4, severe rejection, showing heavier lymphocytes infiltrates appeared in epidermis with necrosis (long black arrow). Scale bar was $50 \mu \mathrm{m}$. (original magnification: A, $\times$ 40; B-F, $\times 100)$.

sen as the sample source because it was a noninvasive sampling procedure and has previously been shown to correspond to intragraft gene expression (Vasconcellos et al. 1998).

Our results showed that MHC-I and -II mRNAs expression were up-regulated during AR. One possible explanation was that MHC-II was recognized as T-lymphocytes activation marker (Sheldon and Poulten 2006). When AR occurs, T-lymphocytes were activated and host cellular immunity and humoral immunity were enhanced, which may trigger the greater expression of MHC-II molecules. Wang et al. (1999) also have confirmed that the elevated MHC-I expression may protect T-cells from antibody- and macrophage-mediated deletion and contribute to the maintenance of low CD95 expression and up-regulated CD28 expression on T-cells. The mecha- nism involved in the higher transcription of MHC gene expression may be more complex in AR and needs to be studied further. The density of one specific peptide-MHC complex required a minimum of 200 molecules to allow $\mathrm{T}$ cell recognition and lysis by cytotoxic T lymphocytes (CTL). Therefore, the increased expression levels of MHC-I mRNAs could contribute to the immunological response.

Interestingly, MHC gene expression levels were distributed in a bimodal form (high and low) in the process of AR (Fig. 4). To explain this tendency, we also attempted to investigate the expression of FasL mRNAs, another accepted marker for AR, in PBLs with real time PCR. The results indicated that the FasL mRNAs expression were elevated at day 8 to 10 post-transplant and exceeded its pre-operation levels in AR (data not 

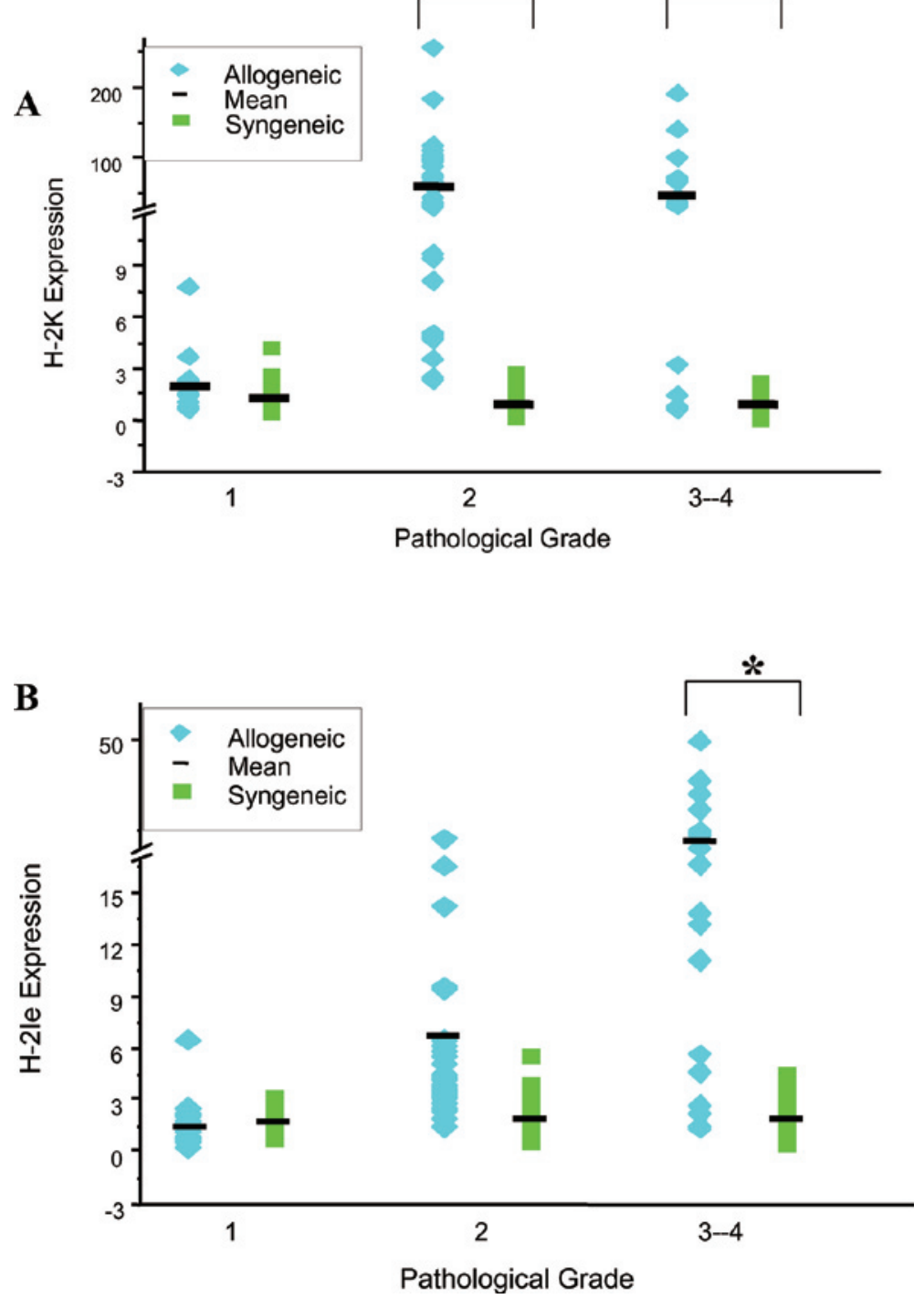

Fig. 3. Expression of H2-K and H-2Ie mRNAs in allogeneic and syngeneic graft group.

The individual gene expression levels were presented in allogeneic and syngeneic graft group. The $\mathrm{x}$-axis showed the pathological rejection grade after transplantation. The $\mathrm{y}$-axis showed the gene expression levels. Both of $\mathrm{H} 2-\mathrm{K}$ and $\mathrm{H}-2 \mathrm{Ie}$ mRNAs levels increased significantly in allograft group compare to syngeneic graft group. ${ }^{*} p<0.05$. A: The increased expression of $\mathrm{H}-2 \mathrm{~K}$ mRNA appeared in acute rejection grade 2-4. B: H-2Ie mRNA level also increased significantly in acute rejection grade $3-4$.

shown). The high expression of FasL mRNAs may contribute to T-lymphocytes apoptosis.

In our study, the expression level of MHC-I (H-2K) mRNA was higher and lasted longer than that of MHC-II (H-2Ie) mRNA, especially during the first peak at day 7 post-transplant. Also the expression of $\mathrm{H}-2 \mathrm{~K}$ mRNA seemed higher than $\mathrm{H}-2 \mathrm{D}$ during the first peak. 61 of $65(93.84 \%)$ allograft samples showed increased level of $\mathrm{H}-2 \mathrm{~K}$ mRNA at post-transplant than at pre-transplant level during AR. Consequently, since the increased levels of MHC-I mRNA, especially $\mathrm{H}-2 \mathrm{~K}$ mRNA, appeared earlier and higher, it may be used as an effective monitoring marker in the primary stage of acute rejection.

It has been demonstrated that some patients 


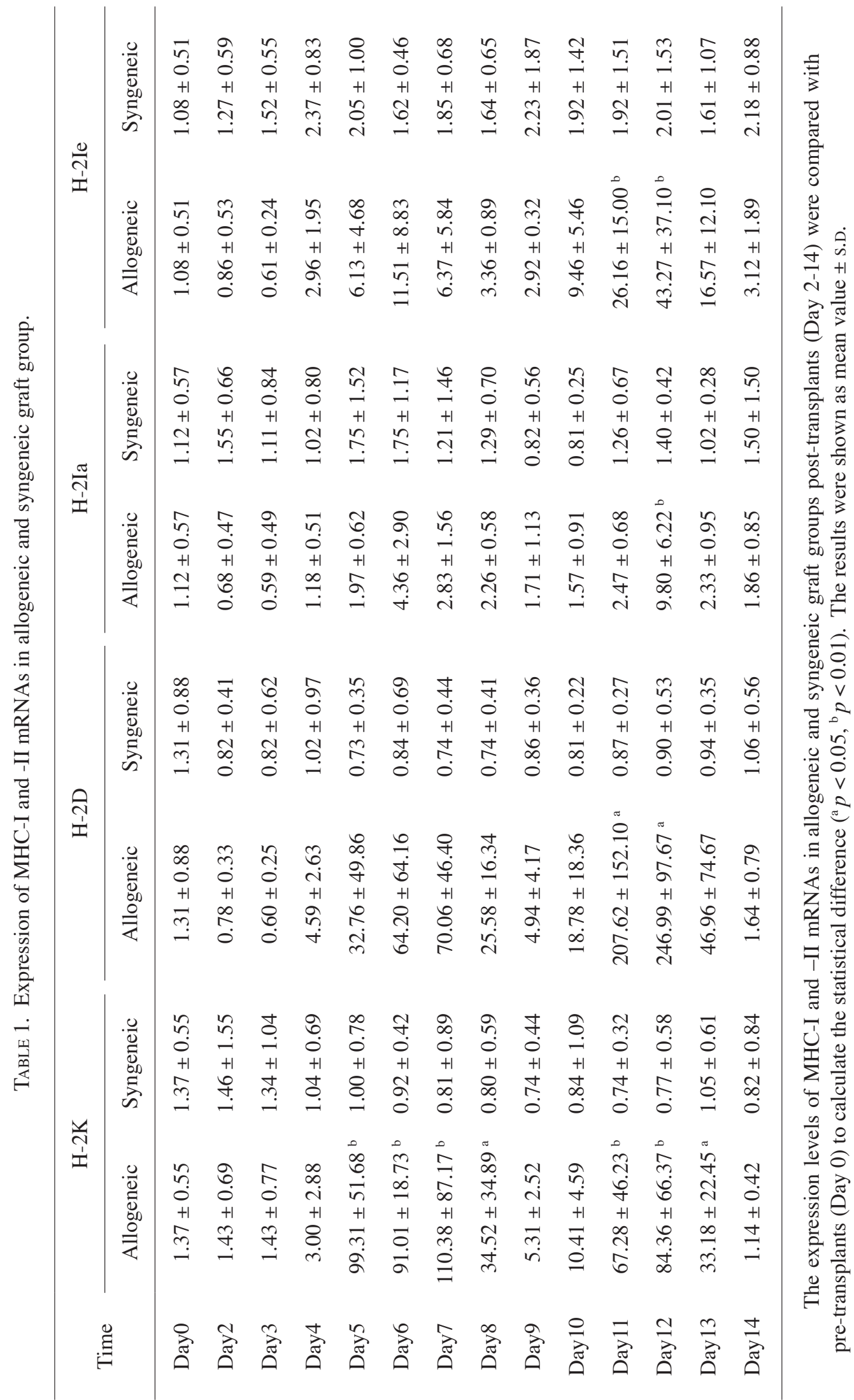



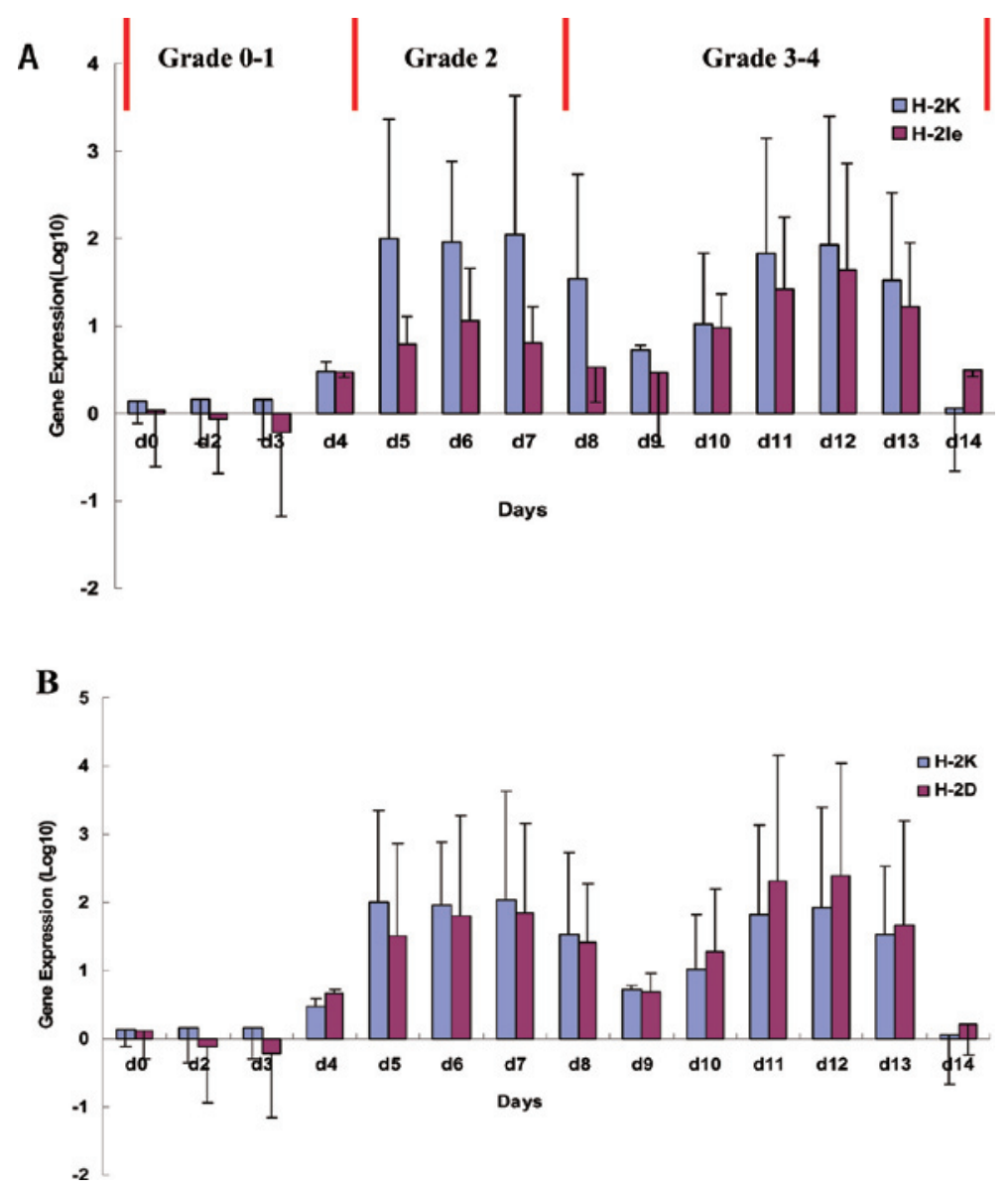

Fig. 4. Expression of MHC-I and -II mRNAs in allograft group.

Results were shown as the means \pm S.D. of gene relative expression levels after skin transplantation. MHC-I and -II mRNAs levels were elevated in the form of bimodal distribution in accordance with AR in allograft group. The relationship between gene expression and pathological rejection grade were shown as day 0 pre-transplant (grade 0), day 2-4 post-transplant (grade 1), day 5-7 post-transplant (grade 2), and day 8-14 post-transplant (grade 3-4). A: The comparison of MHC-I (H-2K) and -II (H-2Ie) mRNAs expression levels. H-2K mRNA level increased significantly at day 5 posttransplant, which appeared earlier than H-2Ie level. B: The comparison of MHC-I, including H-2K and $\mathrm{H}-2 \mathrm{D}$ loci, mRNA expression levels.

without evidence of clinical rejection still exhibited inflammatory and cytotoxic tendencies. This phenomenon was called "subclinical" rejection (Rush 2004). Subclinical rejection may represent a substantial proportion of graft rejection, of which the pathogenicity has not been recognized because of the lack of sensitive detection methods. The biopsy has the drawbacks of invasive risk and cost in the pathological diagnosis of subclinical rejection. Earlier detection of the subclinical rejection would be beneficial to the long-term graft. H-2K mRNA expression in PBLs increased during the primary stage of host immunological response to allograft at day 4 post-transplant, corresponding to pathological rejection grade 1 (Fig. 3 ), and appeared earlier approximately 5 to 6 days than graft rejection observed grossly. Thus, the higher the level of $\mathrm{H}-2 \mathrm{~K}$ mRNA expression at the early stage of acute rejection, the more valuable it will become in the clinical settings.

\section{Acknowledgments}

The Key Laboratory of Cardiovascular Remodeling and Function Research, Chinese Ministry of 
Education and Chinese Ministry of Public Health supported this study.

\section{References}

Bejarano, P.A., Levi, D., Nassiri, M., Vincek, V., Garcia, M. \& Weppler, D. (2004) The Pathology of full-thickness cadaver skin transplant for large abdominal defects: a proposed grading system for skin allograft acute rejection. Am. J. Surg. Pathol., 28, 670-675.

Borelli, S.D., Ferreira, E., Oliveira, A.M., Krishnaswamy, S., Hiraki, D.D. \& Grumet, F.C. (1999) Specific sHLA in healthy donors and donor-specific sHLA in renal transplant patients. Hum. Immunol., 60, 430-434.

Davenport, M., Peakman, M., Dunne, J.B., Gonde, C.E., Vergani, D. \& Williams, R. (1996) Peripheral blood and intrahepatic subsets of T lymphocyte activation and function in liver allograft rejection and drug-induced tolerance in rats. Transpl. Immunol., 4, 126-132.

Schefe, Jan. H., Lehmann, K.E., Buschmann, I.R., Unger, T. \& Funke-Kaiser, H. (2006) Quantitative real-time RT-PCR data analysis: current concepts and the novel "gene expression's C(T) difference" formula. J. Mol. Med., 84, 901-910.

Klein, J., Figueroa, F. \& David, C.S. (1983) H-2 haplotypes, genes and antigens: 2nd listing II. The H-2 complex. Immunogenetics, 17, 553-596.
Mathew, J.M., Shenoy, S., Phelan, D., Lowell, J., Howard, T. \& Mohanakumar, T. (1996) Biochemical and immunological evaluation of donor-specific soluble HLA in the circulation of liver transplant recipients. Transplantation, 62, 217.

Rush, D. (2004) Insights into subclinical rejection. Transpl. Proc., 36, 71-73.

Sabek, O., Dorak, M.T., Kotb, M., Gaber, A.O. \& Gaber, L. (2002) Quantitative detection of T-cell activation markers by real-time PCR in renal transplant rejection and correlation with histopathologic evaluation. Transplantation, $\mathbf{7 4}$ 701-707.

Sheldon, S. \& Poulton, K. (2006) HLA typing and its influence on organ transplantation. Methods Mol. Biol., 333, 157-174.

Silva, F.R., Silva, L.B., Cury, P.M., Burdmann, E.A. \& Bueno, V. (2006) FTY720 in combination with cyclosporine--an analysis of skin allograft survival and renal function. Int Immunopharmacol., 6, 1911-1918.

Vasconcellos, L.M., Schachter, A.D., Zheng, X.X., Vasconcellos, L.H., Shapiro, M. \& Harmon, W.E. (1998) Cytotoxic lymphocyte gene expression in peripheral blood leukocytes correlates with rejecting renal allografts. Transplantation, 66, 562-566.

Wang, Z.Q., Bapat, Abhijit, S. \& Rejo, V. (1999) MHC class I molecules on CD4 T cells regulate receptor-mediated activation signals. Cell Immunol., 193, 108-114. 